\title{
DISTRIBUTION OF AND KEY TO SPECIES OF THE GENUS Daptonema Cobb, 1920 IN VIETNAM
}

\author{
Nguyen Dinh Tu ${ }^{1,2, *}$, Nguyen Vu Thanh ${ }^{1}$ \\ ${ }^{1}$ Institute of Ecology and Biological Resources, VAST, Vietnam \\ ${ }^{2}$ Graduate University of Science and Technology, VAST, Vietnam
}

Received 27 August 2018, accepted 15 February 2019

\begin{abstract}
Up to now, twenty two nematodes species were recognized in the genus Daptonema Cobb, 1920 from mangrove, river and estuary ecosystems in Vietnam, of those, eighteen species were from Vietnam's nematode fauna. Three species, D. dihystera Gagarin and Nguyen Vu Thanh, 2005, D. salvum Gagarin, Nguyen Vu Thanh \& Nguyen Thi Thu, 2005 and D. rigidum Gagarin, Nguyen $\mathrm{Vu}$ Thanh \& Nguyen Thi Thu, 2005 were found in fresh water, while other species were distributed in the mangrove forest, estuaries and seagrass ecosystem. The distribution and taxonomic keys to the already known species of the genus Daptonema Cobb, 1920 in Vietnam was provided.
\end{abstract}

Keywords: Nematodes, Daptonema, distribution, taxonomy, Vietnam.

Citation: Nguyen Dinh Tu, Nguyen Vu Thanh. Distribution of and key to species of the genus Daptonema Cobb, 1920 in Vietnam. Tap chi Sinh hoc, 41(1): 1-8. https://doi.org/10.15625/0866-7160/v41n1.13016.

*Corresponding author email: ngdtu@yahoo.com

(C2019 Vietnam Academy of Science and Technology (VAST) 


\title{
PHÂN BỐ VÀ KHOÁ ĐỊNH LOẠI CÁC LOÀI TUYẾN TRÙNG THUỘC GIỐNG Daptonema Cobb, 1920 Ở VIẸT NAM
}

\author{
Nguyễn Đình Tứ ${ }^{1,2, *}$, Nguyễn Vũ Thanh ${ }^{1}$ \\ ${ }^{1}$ Viện Sinh thái và Tài nguyên Sinh vật, Viện Hàn lâm Khoa học và Công nghệ Việt Nam, Việt Nam \\ ${ }^{2}$ Học Viện Khoa học và Công nghệ, Viện Hàn lâm Khoa học và Công nghệ Việt Nam, Việt Nam
}

Ngày nhận bài 27-8-2018, ngày chấp nhận 18-2-2019

\section{TÓM TÁ̀T}

Daptonema Cobb, 1920 là một giống tuyến trùng biển, đây là một giống lớn, cho đến nay khu hệ thế giới có khoảng 117 đến 136 loài chính thức được thừa nhận và được phát hiện. Ở rừng ngập mặn và biển ven bờ Việt Nam, chúng tôi đã phát hiện được 22 loài tuyến trùng thuộc giống Daptonema. Trong đó, có 18 loài mới cho khoa học đã được mô tả từ khu hệ của Việt Nam. Có 3 loài phân bố ở thuỷ vực nước ngọt là $D$. dihystera Gagarin and Nguyen Vu Thanh, 2005; $D$. salvum Gagarin, Nguyen Vu Thanh \& Nguyen Thi Thu, 2005 và D. rigidum Gagarin, Nguyen Vu Thanh \& Nguyen Thi Thu, 2005; những loài còn lại có phân bố rải rác ở các hệ sinh thái cửa sông, rừng ngập mặn và cỏ biển. Trong bài báo này, chúng tôi đã xây dựng khoá định loại bằng hình ảnh của 22 loài tuyến trùng thuộc giống Daptonema ở Việt Nam.

Từ khóa: Daptonema, tuyến trùng, phân bố, khóa phân loại, Việt Nam.

*Địa chỉ liên hệemail: ngdtu@yahoo.com

\section{MỞ ĐẦU}

Trong nhiều năm trở lại đây, phòng Tuyến trùng học, Viện Sinh thái và Tài nguyên Sinh vật đã có nhiều công bố về sinh thái học, phân loại học và mô tả các loài tuyến trùng mới cũng như các loài đã phát hiện được ở các hệ sinh thái nước ngọt và nước mặn của Việt Nam. Đặc biệt, đã có tới 22 loài ghi nhận và loài mới thuộc giống Daptonema Cobb, 1920 ở vùng nước ngọt, nước lợ và nước mặn của Việt Nam trong (Nguyen Dinh $\mathrm{Tu}$ et al., 2018). Daptonema là một giống tuyến trùng biển rất lớn, với khoảng 117 đến 136 loài chính thức được thừa nhận và được phát hiện trên toàn thế giới (Venekeyetal, 2014; Fonseca \& Bezerra, 2014; Gagarin, 2018). Trong bài báo này, chúng tôi hệ thống 22 loài tuyến trùng thuộc giống Daptonema ở Việt Nam đồn thời xây dựng khoá định loại lưỡng phân và khoá định loại bằng hình ảnh các loài tuyến trùng của Việt Nam.

\section{VÂT LIỆ VÀ PHƯƠNG PHÁP NGHIÊN CƯU}

Các mẫu bùn được thu thập bằng gầu đáy Ponar tại một số hệ sinh thái chủ yếu của rừng ngập mặn (Rừng ngập mặn Tiên Yên, tỉnh Quảng Ninh; rừng ngập mặn $\mathrm{Ba}$ Lạt, tỉnh $\mathrm{Nam}$ Định và Thái Bình; rừng ngập mặn Cần Giờ, thành phố Hồ Chí Minh; sông và cửa sông sông lớn của Việt Nam (sông Chu, Thanh Hoá, sông Đồng Nai, tỉnh Đồng Nai, hệ thống sông Mê Kông).

Mẫu tuyến trùng sau khi thu được cố định bằng dung dịch formalin nóng $5 \%$. Tuyến trùng được tách chiết bằng phương pháp $\mathrm{ly}$ tâm theo De Grisse (1969), được làm trong bằng glycerine (Seinhorst, 1959) và được lên tiêu bản để phân loại trên kính hiển vi.

Khoá định loại được xây dựng theo những nguyên tắc sau:

Đặc điểm phải đặc trưng cho mọi cá thể của loài, tương đối ổn định, có thể quan sát 
chúng một cách dễ dàng, đặc biệt, những đặc điểm là các số đo, các chỉ số liên quan.

Lập bảng thống kê các đặc điểm của loài, gồm những đặc điểm đặc trưng và khác nhau giữa các loài trong cùng một giông.

Trong bài báo này, chúng tôi tham khảo những tài liệu mô tả các loài tuyến trùng đã được công bố từ trước tới nay. Xây dựng hoá định loại lưỡng phân và khoá định loại bằng hình ảnh của các loài tuyến trùng thuộc giống Daptonema ở Việt Nam.

Trong khóa định loại, sử dụng đặc điểm dễ nhận biết về hình thái như: phân bố ở các thuỷ vực khác nhau, chiều dài cơ thể, chiều dài lông đầu, tỉ lệ \% chiều dài lông đầu so với chiều rộng cơ thể tại vị trí gốc lông đầu, các chỉ số $a, b, c$ ', chiều dài và hình dạng của gai sinh dục, chiều dài và hình dạng của đuôi.

\section{KẾT QUẢ VÀ THẢO LUẬN}

\section{Giống Daptonema Cobb, 1920}

\section{Hệ thống phân loại học}

Ngành Nematoda Potts, 1932

Lớp Chromadorea Inglis, 1983

Bộ Monhysterida Filipjev, 1929
Họ Xyalidae Chitwood, 1951

Giống Daptonema Cobb, 1920

\section{Đặc điểm chẩn loại}

Cutin phân đốt, trên bề mặt vỏ cutin thường có rất nhiều các lông dinh dưỡng (somatic), chiều dài của các lông này không vượt 1 lần đường kính cơ thể. Lông cứng ở đầu thường có 10-14 chiếc và phân bố thành 6 nhóm. Xoang miệng hình chóp, đôi khi dạng phễu và không có các trang điểm khác, Cố thường có các lông cứng. Các mắt sắc tố không có (ocelli). Thực quản hình trụ với phần gốc thực quản (đoạn nối thực quản với ruột) không phình. Gai sinh dục của con đực ngắn, nhỏ hơn 2 lần a.b.d (đường kính cơ thể tại lỗ huyệt) và cong, trợ gai có thể có hoặc không. Trên mút đuôi bao giờ cũng có 2-3 lông cứng.

Trong số 22 loài Tuyến trùng thuộc giống Daptonema có 3 loài phân bố ở thuỷ vực nước ngọt là $D$. dihystera Gagarin \& Nguyen $\mathrm{Vu}$ Thanh, D. salvum Gagarin, Nguyen Vu Thanh $\&$ Nguyen Thi Thu và $D$. rigidum Gagarin, Nguyen Vu Thanh \& Nguyen Thi Thu, những loài còn lại phân bố rải rác ở các hệ sinh thái cửa sông, rừng ngập mặn và cỏ biển (bảng 1 ).

Bảng 1 . Thành phần loài và phân bố của các loài tuyến trùng thuộc giống Daptonema Cobb, 1920 ở Việt Nam

\begin{tabular}{|c|l|l|}
\hline TT & \multicolumn{1}{|c|}{ Tên loài } & \multicolumn{1}{|c|}{ Phân bố } \\
\hline 1 & $\begin{array}{l}\text { D. balatum Nguyen Dinh Tu, Gagarin, Nguyen } \\
\text { Vu Thanh, Nguyen Thi Xuan Phuong \& Nguyen } \\
\text { Thanh Hien, 2014. }\end{array}$ & $\begin{array}{l}\text { Rừng ngập mặn Ba Lạt, Nam } \\
\text { Định }\end{array}$ \\
\hline 2 & $\begin{array}{l}\text { D. brevisetosum Nguyen Vu Thanh \& Gagarin, } \\
\text { 2009 }\end{array}$ & Đồng bằng Sông Cửu Long \\
\hline 3 & D. communis Gagarin \& Nguyen Vu Thanh, 2014 & $\begin{array}{l}\text { Rừng ngập mặn Tiên Yên, Quảng } \\
\text { Ninh }\end{array}$ \\
\hline 4 & D. dihystera Gagarin t Nguyen Vu Thanh, 2005 & Sông Nhuệ, Hà Nội \\
\hline 5 & $\begin{array}{l}\text { D. dolichurum Nguyen Thi Thu, Nguyen Vu } \\
\text { Thanh \& Gagarin, 2004 }\end{array}$ & $\begin{array}{l}\text { Rừng ngập mặn Cần Giờ, thành } \\
\text { phồ Hồ Chính; Rừng ngập } \\
\text { mặn Ba Lạt, Nam Định }\end{array}$ \\
\hline 6 & D. durum Gagarin \& Nguyen Thi Thu, 2008 & Rừng ngập mặn Ba Lạt, Nam Định \\
\hline 7 & D. elongatum Gagarin \& Nguyen Thi Thu, 2008 & Rừng ngập mặn Ba Lạt, Nam Định \\
\hline 8 & D. foetidum Gagarin \& Nguyen Vu Thanh, 2010 & Sông Hàm Luông, Bến Tre \\
\hline 9 & D. gracilima Nguyen Vu Thanh \& Gagarin, 2004 & Rừng ngập mặn Cần Giờ, thành \\
\hline
\end{tabular}




\begin{tabular}{|c|c|c|}
\hline & & phố Hồ Chí Minh \\
\hline 10 & D. hirtum Gerlach, 1951 & Sông Cửu Long, Bến Tre \\
\hline 11 & $\begin{array}{l}\text { D. iners Nguyen Thi Thu, Nguyen Vu Thanh \& } \\
\text { Gagarin, } 2004\end{array}$ & $\begin{array}{l}\text { Rừng ngập mặn Cần Giờ, thành } \\
\text { phố Hồ Chí Minh }\end{array}$ \\
\hline 12 & D. mekongi Nguyen Vu Thanh \& Gagarin, 2004 & $\begin{array}{l}\text { Rừng ngập mặn Cần Giờ, thành } \\
\text { phố Hồ Chí Minh }\end{array}$ \\
\hline 13 & D. obesum Gagarin, 2013 & $\begin{array}{l}\text { Rừng ngập mặn Cần Giờ, thành } \\
\text { phố Hồ Chí Minh }\end{array}$ \\
\hline 14 & D. orientale Gagarin \& Nguyen Vu Thanh, 2005 & $\begin{array}{l}\text { Rừng ngập mặn Ba Lạt, Nam } \\
\text { Định }\end{array}$ \\
\hline 15 & $\begin{array}{l}D \text { (Theristus). oxyuroides (Stekhoven, 1931) } \\
\text { Gagarin \& Lemzina, } 1981\end{array}$ & Sông Cấm, Hải Phòng \\
\hline 16 & $\begin{array}{l}\text { D. paraoxyuroides Nguyen Dinh Tu, Gagarin, } \\
\text { Nguyen Vu Thanh, Nguyen Thi Xuan Phuong \& } \\
\text { Nguyen Thanh Hien, } 2014\end{array}$ & $\begin{array}{l}\text { Rừng ngập mặn Ba Lạt, Nam } \\
\text { Định; Rừng ngập mặn Tiên Yên, } \\
\text { Quảng Ninh }\end{array}$ \\
\hline 17 & $\begin{array}{l}\text { D. pumilum Nguyen Vu Thanh, Lai Phu Hoang \& } \\
\text { Gagarin, } 2005\end{array}$ & Sông Cấm, Hải Phòng \\
\hline 18 & $\begin{array}{l}\text { D. rigidum Gagarin, Nguyen Vu Thanh \& Nguyen } \\
\text { Thi Thu, } 2005\end{array}$ & Sông Chu, Thanh Hoá \\
\hline 19 & $\begin{array}{l}\text { D. salvum Gagarin, Nguyen Vu Thanh \& Nguyen } \\
\text { Thi Thu, } 2005\end{array}$ & Sông Chu, Thanh Hoá \\
\hline 20 & $\begin{array}{l}\text { D. securum Nguyen Thi Xuan Phuong, Pham Thi } \\
\text { Man, Nguyen Dinh Tu, } 2016\end{array}$ & Cát Bà, Hải Phòng \\
\hline 21 & D. setosum (Bütschli, 1874) & $\begin{array}{l}\text { Rừng ngập mặn Ba Lạt, Nam } \\
\text { Định; Rừng ngập mặn Tiên Yên, } \\
\text { Quảng Ninh }\end{array}$ \\
\hline 22 & $\begin{array}{l}\text { D. vietnamensis (Gagarin \& Thu, 2008) Venekey, } \\
\text { Gheller, Maria, Brustolin, Kandratavicius, Vieira, } \\
\text { Brito, Souza, Fonseca, } 2014\end{array}$ & Rừng ngập mặn Ba Lạt, Nam Định \\
\hline
\end{tabular}

Khoá định loại các loài thuộc giống Daptonema Cobb, 1920

1a. Sống ở môi trường nước ngọt.......................................................

b. Sống ở môi trường nước lợ và nước mặn............................................ 4

2a. Gai sinh dục mảnh, cu tin hoá yếu, tương đối thẳng........................... rigidum

b. Gai sinh dục dày, cu tin hoá mạnh, không có cấu trúc thẳng.............................

3a. Gai sinh dục có cấu tạo vuông góc tại vị trí $1 / 2$ từ gốc của gai sinh dục, trợ gai có mấu

b. Gai sinh dục có cấu tạo vuông góc tại vị trí $2 / 3$ từ gốc của gai sinh dục.....D. dinhystera

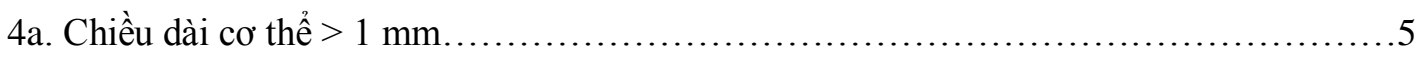

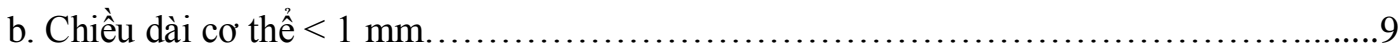

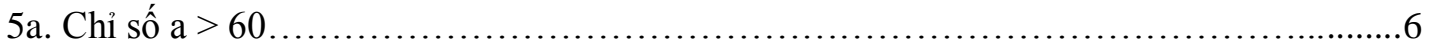

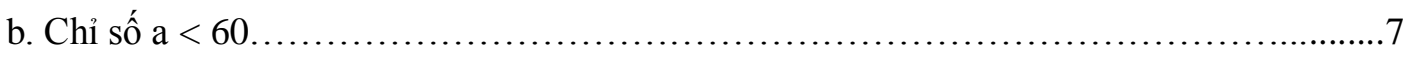


6a. Tỉ lệ \% chiều dài lông đầu so với chiều rộng cơ thể tại vị trí gốc lông đầu $>80$.

D. dolichurum

b. Tỉ lệ \% chiều dài lông đầu so với chiều rộng cơ thể tại vị trí gốc lông đầu < 80

D. gracilimia

7a. Chỉ số b $>7$. D. brevisetosum

b. Chỉ số $b<7$ .8

8a. Chiều dài gai sinh dục $>40 \mu \mathrm{m}$ D. setosum

b. Chiều dài gai sinh dục $<40 \mu \mathrm{m}$ D. oxyuroides

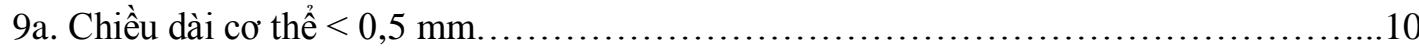

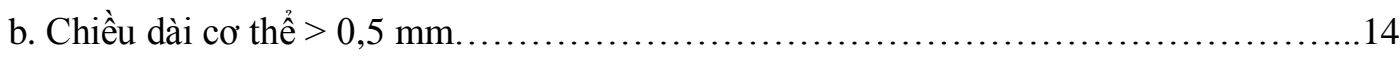

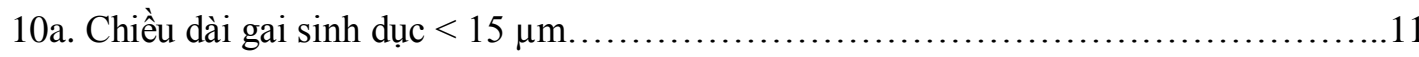

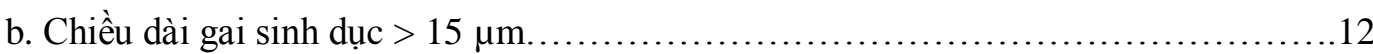

11a. Tỉ lệ \% chiều dài lông đầu so với chiều rộng cơ thể tại vị trí gốc lông đầu > 70 .

D. durum

b. Tỉ lệ \% chiều dài lông đầu so với chiều rộng cơ thể tại vị trí gốc lông đầu < 70 .

D. pumilum

c. Chiều dài lông đầu $>5 \mu \mathrm{m} \ldots \ldots \ldots \ldots \ldots \ldots \ldots \ldots \ldots \ldots . \ldots \ldots$. vietnamensis (curvatum)

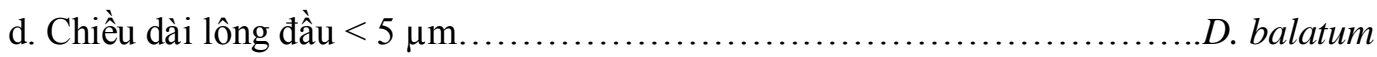

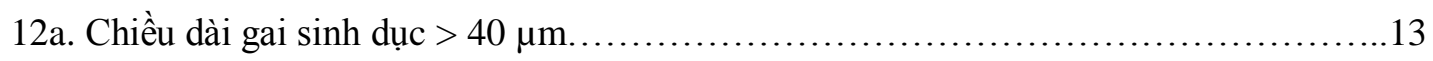

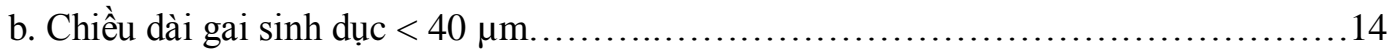

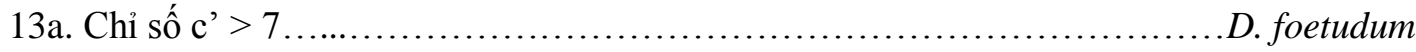

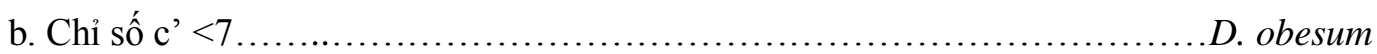

14a. Tỉ lệ \% chiều dài lông đầu so với chiều rộng cơ thể tại vị trí gốc lông đầu > 50........15

b. Tỉ lệ \% chiều dài lông đầu so với chiều rộng cơ thể tại vị trí gốc lông đầu < 50........19

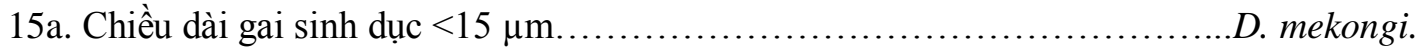

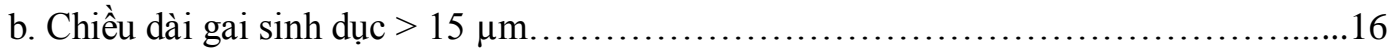

16a. Tỉ lệ \% chiều dài lông đầu so với chiều rộng cơ thể tại vị trí gốc lông đầu bằng $100 \ldots \ldots$.... D. securum

b. Tỉ lệ \% chiều dài lông đầu so với vị trí chiều rộng cơ thể tại lông đầu <80 17

17a. Gai sinh dục mảnh, cu tin hoá yếu, có hình lòng máng. D. elongatum

b. Gai sinh dục dày, cu tin hoá mạnh, không có cấu trúc lòng máng. . .18

18a. Trợ gai to, vuông góc với gai sinh dục, gai sinh dục có cấu tạo vuông góc tại vị trí $2 / 3$ tính từ gốc gai sinh dục

D. histum

b. Trợ gai sinh dục có hình ống, gai sinh dục có cấu tạo vuông góc tại vị trí 1/3 tính từ gốc gai sinh dục.

D. communis 
19a. Đuôi dài, dạng chỉ, lông đầu rất dài, chiếm $45 \%$ tại vị trí chiều rộng cơ thể tại gốc lông đầu. D. iners

b. Đuôi dạng chóp tù, lông đầu ngắn, tỉ lệ \% chiều dài lông đầu so với vị trí chiều rộng cơ thể tại gốc lông đầu $<40 \%$

20a. Gốc gai sinh dục loe tròn, trợ gai vuông góc với gai sinh dục về phía đuôi D. orientale

b. Gai sinh dục mảnh, trợ gai bé, song song với gai sinh dục .D. paraoxyuroides

Hình thái các loài thuộc giống Daptonema Cobb, 1920

Khóa định loại bằng hình ảnh các loài Tuyến trùng thuộc giống Daptonema Cobb, 1920 phân bố Việt Nam được xây dựng dựa trên các hình vẽ của các tác giả trong công bố của mình. Để thuận tiện cho việc sử dụng hình ảnh để hỗ trợ trong quá trình phân loại, chúng tôi đưa ra hình ảnh của mỗi loại bao gồm phần đầu (cấu trúc amphid, lông đầu, khoang miệng...) và phần đuôi (bao gồm gai sinh dục, cấu trúc của đuôi...). Khoá định loại bằng hình ảnh được thể hiện như sau:

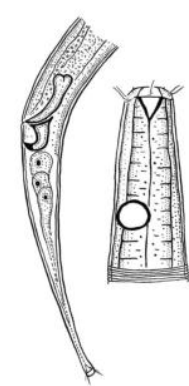

D. blatum

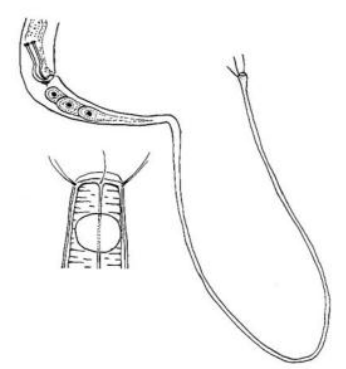

D. dolichurum

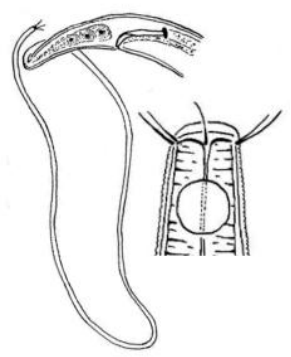

D. gracilima

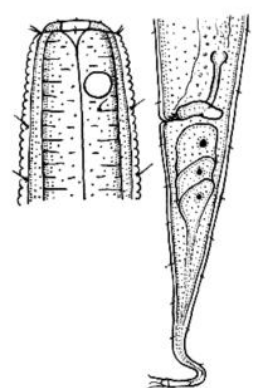

D. brevisetosum

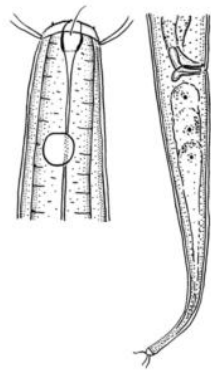

D. durum

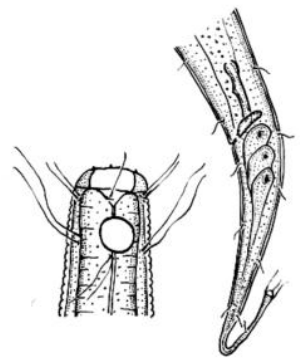

D. histum

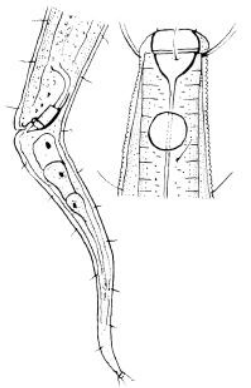

D. communis

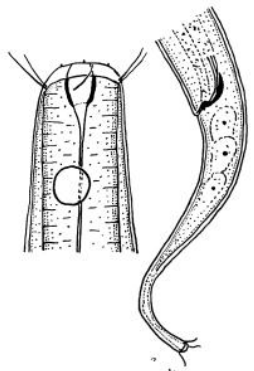

D. elongatum

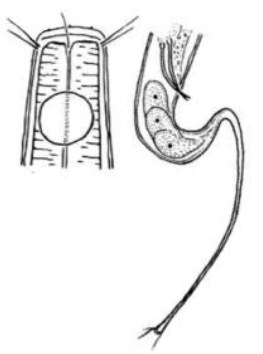

D. iners

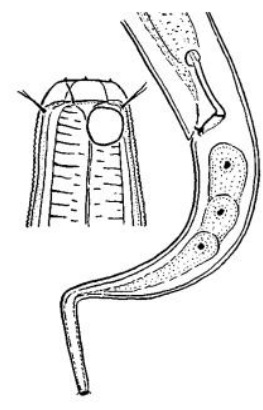

D. dinhystera

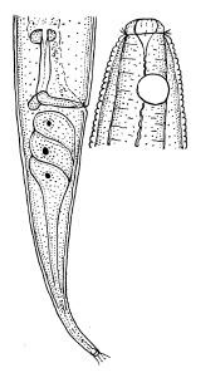

D. foetidum

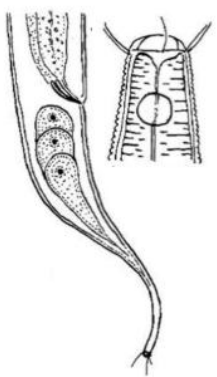

D. mekongi 


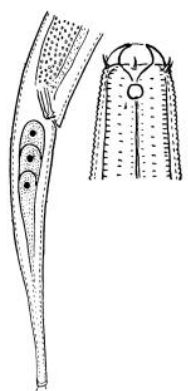

D. obesum

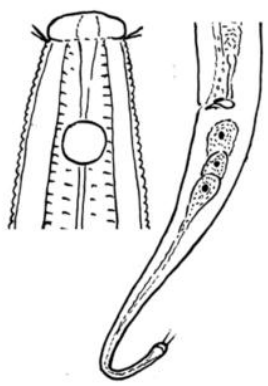

D. pumilum

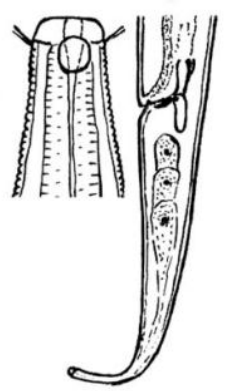

D. orientale
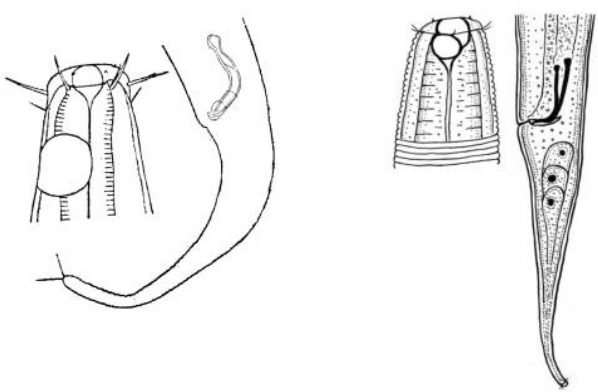

D. oxyuroides

D. paraoxyuroides
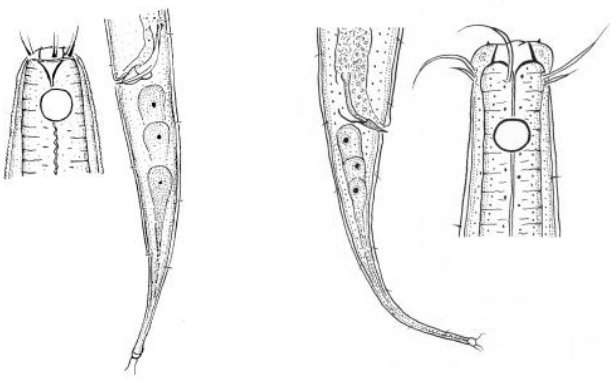

D. salvum

D. securum

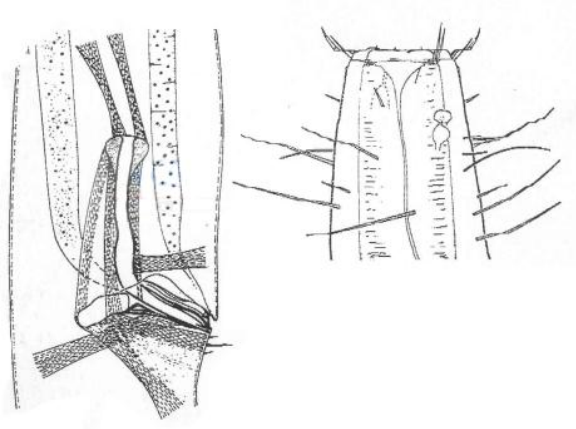

D. setosum

Lời cảm ơn: Nghiên cứu này được tài trợ bởi Quỹ Phát triển khoa học và công nghệ Quốc gia (NAFOSTED) trong đề tài mã số: FWO.106-NN.2015.04.

\section{TÀI LIỆU THAM KHẢO}

Bütschli O., 1874. Zur Kenntnis der freilebenden Nematoden, insbesondere der des Kieler Hafens. Abhandl. d. Senckenb. naturf. Gesellsch. IX. Bd.: 1-56.

Tài liệu tham khảo không đánh số viết như tài liệu trên.Gagarin V. G., 2001. New species of free-living nematodes from Biwa Lake and inflowing stream Honshu

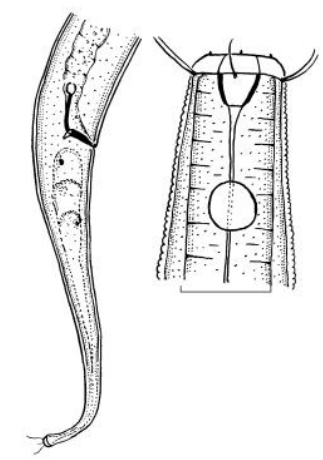

D. vietnamensis (curvatum)

Island, Japan. Zoologicheskii Zhurnal, 80(1): 12-25.

Gagarin V. G., 2013. Daptonema obesum sp. $\mathrm{n}$. and Steineria vietnamica $\mathrm{sp}$. $\mathrm{n}$. (Nematoda, Xyalidae) from mangrove forest of Vietnam. International Journal of Nematology, 23(2): 129-137.

Gagarin V. G., 2018. An annotated checklist of the free-living nematodes from mangrovethickets of Vietnam. Zootaxa 4403, (20): 261-288.

Gagarin V. G., Nguyen Thi Thu, 2008. Three new species of the genus Daptonema 
(Nematoda, Xyalidae) from the Red river delta (Vietnam). Zoologicheskii Zhurnal, 87(5): 515-523.

Gagarin V. G., Nguyen V. T., 2012. Freeliving nematodes of Tra Ly river in the Red river mouth, Vietnam. Inland Water Biology, 5: 11-18.

Gagarin V. G., Nguyen Vu Thanh, 2007. Free-living nematodes of the Mekong Delta (Vietnam). Biology of Inland Waters, 3: 3-10.

Gagarin V. G., Nguyen Vu Thanh, 2010. Two new species of the family Xyalidae Chitwood, 1951 (Nematoda, Monhysterida) from littoral zone of South China Sea. International Journal of Nematology, 20(1): 1-6.

Gagarin V. G., Nguyen Vu Thanh, 2014. Two new species of the family Xyalidae Chitwood, $1951 \quad$ (Nematoda, Monhysterida) from the coast of Vietnam. International Journal of Nematology, 24(2): 108-116.

Gagarin V. G., Nguyen Vu Thanh, Nguyen Thi Thu, 2005. Daptonema salvum sp. $\mathrm{n}$. and $D$. rigidum sp. n. (Nematoda: Monhysterida) from Chu River, Vietnam. International Journal of Nematology, 24(2): 210-214.

Gagarin V. G., Thanh N. V., 2009. Three new species of Monhysterids (Nematoda, Monhysterida) from a mangrove forest in the Mekong River Delta, Vietnam. Zoosyst Rossica, 88(10): 1170-1178.

Gerlach S., $1965 . \quad$ Freilebende Meeresnematoden aus der Gezeitenzone von Spizbergen // Veroffentlichungen des Instituts fur Meeresforschungen in Bremerhaven. Bd.9. Heft 2. S. 109-172.

Nguyen D. T., Gagarin V. G., Nguyen V. T., Nguyen T. X. P., Nguyen T. H., 2014. Two new nematode species of the genus Daptonema Cobb, 1920 (Nematoda, Xyalidae) from mangrove forest estuary of the Red river (Vietnam). Inland Water Biology, 7: 125-133.
Nguyen Dinh $\mathrm{Tu}$, Nguyen Thanh Hien, Gagarin V. G., Nguyen Vu Thanh, 2018. The investigating situation of marine nematodes of the genus Daptonema Cobb, 1920 in mangrove and estuary ecosystems of Vietnam coastal sea. Proceedings of the $5^{\text {th }}$ International Scientific and Technical Conference. Vladivostok, 22-24 May. 220-227.

Nguyen Thi Thu, Nguyen Vu Thanh, Gagarin V. G., 2004. Two new brackish water nematode species of the genus Daptonema Cobb, 1920 (Nematoda: Monhysterida) from Can Gio mangrove. National Conference of Life Sciences Thai Nguyen University. 249-252.

Nguyen Thi Xuan Phuong, Judith C. K., Pham Thi Man, Gagarin V. G., Nguyen Dinh Tu., 2016. Description of the new species of free-livings nematodes Daptonema securum sp. nov. from artificial reservoirs in Vietnam. Amurian Zoological Journal, 8(4): 225-232.

Nguyen Vu Thanh, Gagarin V. G., 2004. Two new free-living brackish water nematode species of the genus Daptonema Cobb, 1920 (Nematoda: Monhysterida) recorded from Can Gio mangrove. National Conference of Life Sciences Thai Nguyen University. 229-232.

Nguyen Vu Thanh, Gagarin V. G., 2009. Three species of monhysterids (Nematoda: Monhysterida) from mangrove forest of the Mekong River Estuary, Vietnam. Tap chi Sinh hoc, 31(2): 8-15.

Nguyen $\mathrm{Vu}$ Thanh, Lai Phu Hoang, Gagarin V. G., 2005. The new species of Daptonema pumilus sp. n. (Nematoda: Monhysterida) in Vietnam. Tap chi Sinh hoc, 27(3): 1-4.

Seinhorst J. W., 1959. A rapid method for the transfer of nematodes from fixative to anhydrous glycerin. Nematologica, 4: 67-69. 